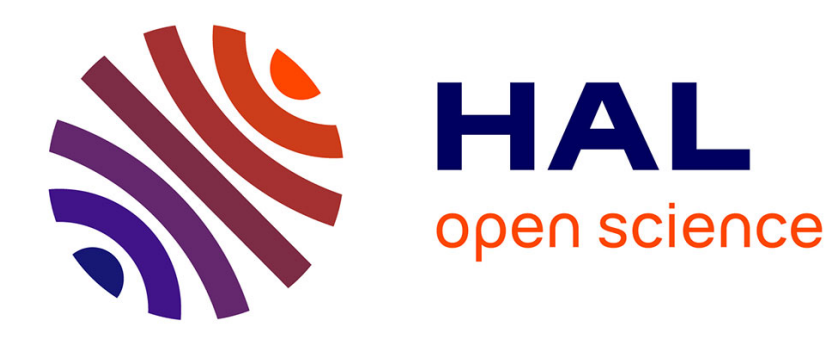

\title{
Gravure et composition métallique des monnaies lagides
}

Thomas Faucher

\section{To cite this version:}

Thomas Faucher. Gravure et composition métallique des monnaies lagides. Revue Numismatique, 2010, pp.95-108. hal-02511074

\section{HAL Id: hal-02511074 \\ https://hal.science/hal-02511074}

Submitted on 18 Mar 2020

HAL is a multi-disciplinary open access archive for the deposit and dissemination of scientific research documents, whether they are published or not. The documents may come from teaching and research institutions in France or abroad, or from public or private research centers.
L'archive ouverte pluridisciplinaire HAL, est destinée au dépôt et à la diffusion de documents scientifiques de niveau recherche, publiés ou non, émanant des établissements d'enseignement et de recherche français ou étrangers, des laboratoires publics ou privés. 


\title{
Gravure et composition métallique des monnaies lagides
}

\begin{abstract}
Résumé - La qualité des graveurs de l'atelier d'Alexandrie pendant la période lagide n'a jamais été remise en cause, même si d'aucuns ont régulièrement taxé le monnayage ptolémaïque de monotonie. De même, les analyses de composition métallique ont très tôt montré que l'aloi des monnaies d'or et d'argent était très bon et ceci pendant une longue période, au moins jusqu'au début du $\mathrm{I}^{\text {er }}$ siècle en ce qui concerne l'argent. Si ce n'est pas tout à fait le cas pour les bronzes ptolémaïques, les analyses effectuées au centre Ernest-Babelon ont mis en lumière la bonne qualité

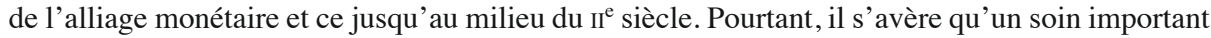
était consacré à la gravure monétaire quand bien même la pièce n'était pas constituée du meilleur alliage. Il est donc naturel de s'interroger sur la corrélation entre la qualité de la gravure et celle de la composition métallique des monnaies.
\end{abstract}

Summary - The skill of the engravers from the mint of Alexandria during the Ptolemaic period is not questionable, even if some charge of monotony the coins of the Ptolemies. Likewise, analyses of metallic content have shown early that the fineness of gold and silver coins is of good quality, and for a long period, at least until the beginning of the first century BC as regards to silver coinage. Even if it is not exactly the case for the bronze currency, analyses held at the Ernest Babelon centre highlight the quality of the monetary alloy until the half of the second century. Though, it turns out that a great care was taken of the engraving even when the coin was not produced with a fine alloy. It is natural to wonder about the correlation between the fineness of the engraving and the one of the coins metallic content.

La matière de cet article est en partie le résultat du travail en collaboration avec J.-N. Barrandon notamment lors d'analyses réalisées à ses côtés au Centre Ernest-Babelon. Il invitait souvent les étudiants à s'interroger sur la pertinence des résultats des analyses et militait toujours pour qu'il existe une interaction entre l'analyste et l'historien ${ }^{1}$. Dans cette perspective, il sera question ici de la corrélation entre la composition métallique des monnaies et la qualité de la gravure des coins, en partant de l'exemple de l'atelier d'Alexandrie à l'époque des Ptolémées.

Le centre Ernest-Babelon a montré un intérêt tout particulier en faveur de la numismatique des Lagides : tout d'abord, il y a quelques années, avec l'analyse de la composition métallique d'un nombre important de monnaies de bronze conservées au Cabinet des Médailles, puis avec l'analyse des monnaies d'or et

* Université Paris-Sorbonne, ANR-Nomisma, 8 rue du Chevalier de La Barre, F-75018 Paris. Courriel : thomas.faucher@paris-sorbonne.fr.

1. On pourra citer à titre d'exemple sa remarquable collaboration avec $\mathrm{O}$. Picard dans l'étude des monnaies antiques de Marseille : J.-N. BarRandon, O. PiCARD, Monnaies de bronze de Marseille, Cahiers Ernest-Babelon, 10, Paris, 2007, 164 p. 
il ne fait pas de doute que la présence de J. Olivier et la préparation de son doctorat sur le monnayage d'argent du $\mathrm{II}^{\mathrm{e}}$ siècle permettront de compléter avantageusement les analyses faites il y a quelques années par R. Hazzard sur les monnaies d'argent et dont nous sommes nombreux à penser qu'elles nécessitent d'être dépoussiérées ${ }^{2}$.

\section{La composition des monnaies lagides}

Il ne s'agit pas ici de présenter l'intégralité des résultats des analyses effectuées au centre Ernest-Babelon, mais plutôt de définir l'évolution globale de la composition métallique des monnaies lagides pour les trois métaux et alliages monétaires utilisés par les Ptolémées : l'or, l'argent et le bronze. Ceci permettra de définir des grandes tendances à l'intérieur desquelles nous pourrons situer les monnaies et comparer par la suite la qualité de leur gravure et leur composition métallique.

Dans le graphique ci-dessous (figure 1), nous avons mis en perspective la composition des monnaies d'or, d'argent et de bronze. Je n'entre pas dans les détails en ce qui concerne le monnayage d'or puisque l'on trouvera dans ce même volume de longs développements à ce sujet dans l'article élaboré par Fr. Duyrat et J. Olivier. Les données reprises pour l'or sont celles obtenues au Centre Ernest-Babelon par J. Olivier dans le cadre de son mémoire de Master.

Sur ce graphique, les données affichées des teneurs en métal fin dans les monnaies d'argent sont issues des deux articles de R. Hazzard qui reprenaient les analyses effectuées par différents chercheurs au cours du siècle dernier ${ }^{3}$.

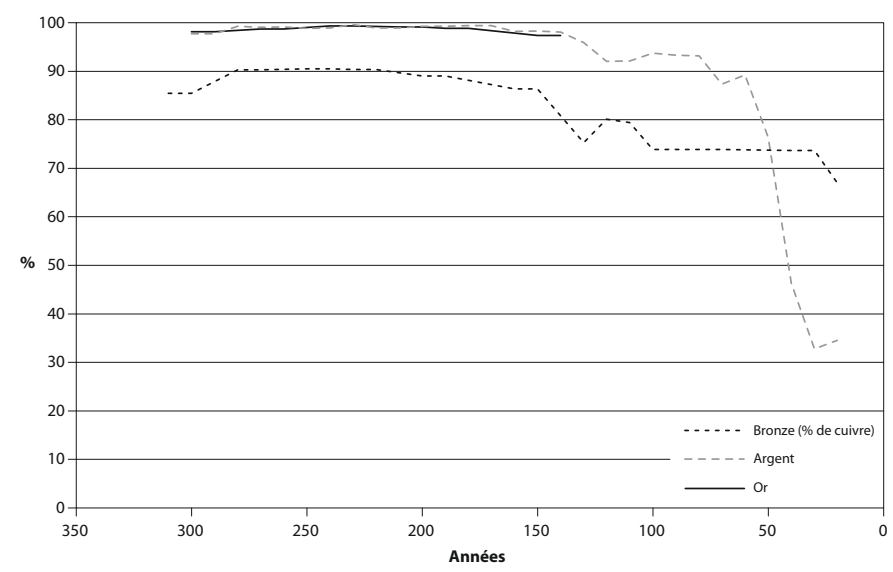

Figure 1 - Évolution de l'alliage monétaire du numéraire lagide.

2. R. HAZZARD, D.I. Brown, The silver standard of the Ptolemaic coinage, RN, 1984, p. 231-239 ; R. HAZZARD, The composition of Ptolemaic silver, Journal of the Society for the Study of Egyptian Antiquities, 1990, p. 89-99.

3. Cf. note précédente. 
Il est difficile de se fier aux résultats de ces analyses tant une multitude de méthodes ont été utilisées par les différents savants : analyses chimiques en phase liquide, activation neutronique, protonique et fluorescence $X$. Ces méthodes sont plus ou moins fiables, la moins fiable étant la fluorescence $\mathrm{X}$ dont on connaît désormais bien les limites. D'ailleurs, R. Hazzard ne s'y est pas trompé ; lorsqu'il dessine la courbe d'évolution de la teneur en argent des monnaies, le trait suit principalement les analyses chimiques et celles par activation, délaissant les analyses, pourtant largement majoritaires, effectuées à la Fluorescence $\mathrm{X}^{4}$.

Les analyses sur le monnayage de bronze ont été réalisées dans le cadre de ma thèse de doctorat, celle-ci portant sur la fabrication des monnaies de l'atelier d'Alexandrie sous les Lagides ${ }^{5}$. Au total, ce sont 128 monnaies du Cabinet des Médailles qui ont ainsi été analysées par ANRC (Activation aux Neutrons Rapides de Cyclotron) au Centre Ernest-Babelon (125 lagides et 3 monnaies d'Auguste de la première série). Les monnaies en bronze ont été reclassées récemment par O. Picard, non plus par règnes, comme on avait coutume de le faire auparavant, mais par séries, définies par le jeu des types du droit et du revers ${ }^{6}$.

Sur le second graphique (figure 2) présentant l'évolution des différents métaux composant la monnaie de bronze, on peut voir, de manière assez sensible, la dégradation de l'alliage au fur et à mesure des séries, le taux d'étain restant relativement stable, le plomb remplaçant de plus en plus le cuivre ${ }^{7}$.

La courbe présente une rupture lors de la huitième série. Il ne faut pas accorder trop d'importance à cette dernière. D'une part, nous n'avons qu'une seule analyse pour caractériser cette série particulière. D'autre part, il s'agit d'une série portant une date au revers, émise sous Ptolémée IX (lors des années 3 et 4 de son règne), dont les monnaies ne se retrouvent pas mélangées à d'autres monnaies dans les trésors ${ }^{8}$. Il se peut qu'il n'y ait pas eu de rappel des anciennes monnaies pour frapper ces pièces, ce qui pourrait expliquer le taux relativement bas de plomb dans l'échantillon. On notera enfin l'accroissement sensible du pourcentage d'étain dans les monnaies frappées par la grande Cléopâtre.

4. R. HAZZARD, The composition..., op. cit. n. 2, p. 92.

5. Th. FAUCHER, L'atelier monétaire d'Alexandrie sous les Lagides : problèmes techniques et stylistiques, thèse soutenue à l'Université Paris-Sorbonne le 9 décembre 2006.

6. O. PicARD, Fr. Duyrat (éd.), L'exception égyptienne? : production et échanges monétaires en Égypte hellénistique et romaine : actes du colloque d'Alexandrie, 13-15 avril 2002. Études alexandrines, 10, Le Caire, 2005 ; Catalogue en cours de publication des monnaies des fouilles du CEAlex.

7. Il est possible que cette utilisation du plomb dans l'alliage monétaire se retrouve dans des alliages utilisés pour la sculpture ou d'autres objets issus de l'artisanat. Le plomb permet à l'alliage de se fluidifier et ainsi d'être utilisé plus facilement pour les coulées lors de la préparation des flans. Des études restent à faire sur ce sujet pour définir si la tendance générale, à partir du II ${ }^{\mathrm{e}}$ siècle, ne va pas dans le sens d'une utilisation accrue du plomb.

8. Th. Faucher, M. Shahin, Le trésor de Gézéïr (Lac Mariout, Alexandrie), RN, 2006, p. 135157. 
La méthode de fabrication n'est pas étrangère à ces évolutions. Pour le bronze, le choix de l'alliage monétaire, c'est-à-dire l'utilisation et la proportion de chaque élément majeur (le cuivre, l'étain et, régulièrement, le plomb), a dépendu en partie de la méthode de fabrication des flans. On distingue trois phases technologiques dans la production des monnaies lagides, plus ou moins imbriquées. Ces méthodes de fabrication ne sont pas sans incidence sur la qualité de la gravure, c'est pour cela qu'il semble nécessaire d'y prêter attention.

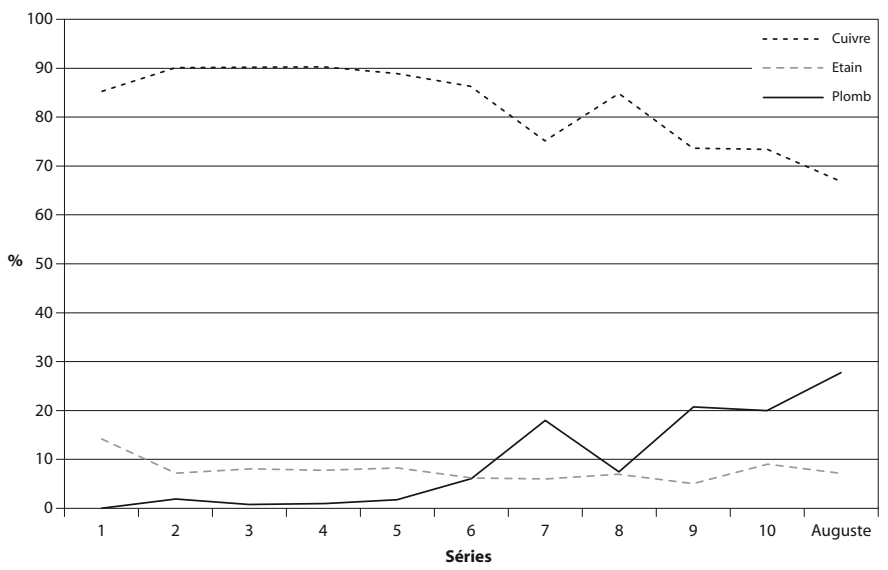

Figure 2 - Évolution des éléments majeurs.

entrant dans la composition des monnaies de bronze lagides.

Sur le graphique, on s'aperçoit que les monnaies de la première série sont caractérisées par un alliage monétaire composé d'un fort taux d'étain et d'une absence de plomb. Les flans de ces monnaies étaient découpés dans des barres de bronze. Après avoir été découpés, ils étaient martelés et recuits pour fournir la forme souhaitée. Cette méthode a été également utilisée pour produire les flans des monnaies de la première partie de la deuxième série.

Pour cette deuxième série, on voit apparaître une autre méthode de fabrication des flans. Ils sont dorénavant obtenus en coulant du métal fondu dans un moule, d'abord à cavités simples, puis en chapelet, comme de nombreux exemples provenant de l'île de Chypre ainsi que de Judée nous le montrent ${ }^{9}$.

9. F. PILON, Un fait unique en Gaule romaine : la découverte de moules à flans monétaires en pierre calcaire, $R S N, 2003$, p. 37-54 ; D. ArIEL, Flan molds from the Temple Mount excavations, dans E. MazAR, The Temple Mount excavations in Jerusalem 1968-1978, Final Report Volume II: The Byzantine and Early Islamic Periods, Jerusalem, 2003, p. 115-119 ; I. Nicolaou, The coins from the House of Dionysos, Nicosie, 1990. 
Les études métallographiques mettent clairement en évidence le changement de technique. Lorsque les monnayeurs commencent à utiliser les moules pour couler leurs flans, les monnaies présentent une structure de grains (base de la structure métallique) de grande taille et de forme arrondie, ce qui suppose que le métal a été coulé puis qu'on l'a laissé refroidir assez lentement ${ }^{10}$. L'emploi de cette technique n'a pas provoqué de changement d'alliage puisque les analyses montrent que les taux d'étain et de cuivre sont restés stables. Cette méthode sera employée par l'atelier officiel jusqu'à la fin du pouvoir lagide mais également par certains faussaires ${ }^{11}$.

Mais, au cours du $\mathrm{II}^{\mathrm{e}}$ siècle, l'Égypte voit apparaître un phénomène nouveau : la production de monnaies moulées. Il s'agit, en partant d'une monnaie officielle, d'obtenir une réplique de la monnaie en utilisant des moules en argile. On connaissait depuis quelques années l'importance de ce fléau dans l'Égypte lagide du I ${ }^{\mathrm{er}}$ siècle $^{12}$. Mais ce n'est que plus récemment que l'on a découvert que ce phénomène touchait également le monnayage de bronze du II ${ }^{\mathrm{e}}$ siècle. On reviendra plus loin sur l'incidence de cette méthode de fabrication sur notre vision du monnayage.
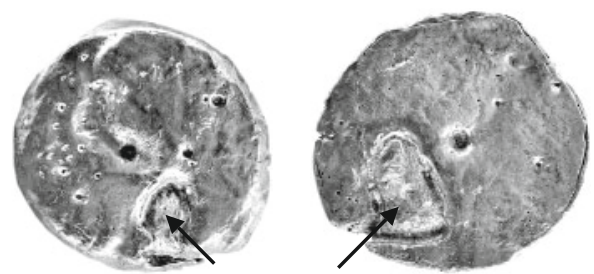

Figure 3 - Monnaie FOUAD 207, CEAlex.
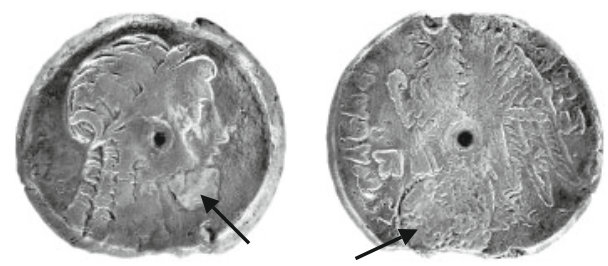

Figure 4 - Monnaie n ${ }^{\circ} 17$ du trésor des bains de Karnak.

10. Th. FAUCHER, L'apport de l'expérimentation à la connaissance des techniques de fabrication des monnaies antiques, dans Artisanat grec : approches méthodologiques et perspectives Actes de la table ronde organisée à Athènes le 5-6 octobre 2007, à paraître. On consultera à ce sujet le livre de D. SCOTT, Metallography and microstructure of ancient and historic metals, M. DEL REY, 1991.

11. Très récemment, un chapelet de flans a été découvert lors des fouilles des chapelles osiriennes de Karnak (chapelle d'Osiris Ounnefer Neb-Djefaou), à l'emplacement probable d'un atelier de faux-monnayeurs.

12. O. PICARD, Monnaies lagides aux deux aigles, $B S F N, 57,2002$, p. 189-191. 
Ci-dessus, sont représentées deux monnaies, l'une est issue des fouilles françaises d'Alexandrie, et l'autre provient du trésor des bains de Karnak, mis au jour fin 2007 dans les fouilles devant le premier pylône du temple de Karnak en Haute Égypte. Ces deux exemplaires laissent entrevoir une partie plus claire, grisée. Il s'agit en fait de nodules de plomb. Le plomb n'étant pas miscible ni avec le cuivre ni avec l'étain, il se trouve dans l'alliage sous forme de nodules qui se sont agglomérés. On ne connaît pas précisément la composition de ces deux monnaies, mais il est probable que leur taux de plomb avoisine les $50 \%{ }^{13}$. C'est un phénomène qu'il est impossible d'observer lors des analyses des monnaies du Cabinet des Médailles, essentiellement parce que ces monnaies de piètre qualité ont été mises au ban des collections. Quoi qu'il en soit, une telle quantité de plomb dans l'alliage n'aurait pas pu permettre à un monnayeur de frapper ce flan : il aurait cassé sous le poids du marteau ${ }^{14}$.

\section{La gravure des monnaies lagides}

On voudrait ici s'intéresser, non pas seulement au savoir faire des graveurs qui ont exercé sur les coins monétaires lagides, mais à la possibilité de mettre en corrélation cette qualité de gravure avec la qualité de la composition métallique étudiée ci-dessus. Pour commencer, juger de la qualité de la gravure n'est pas chose facile, il est en effet légitime de s'interroger sur ce qui détermine la qualité d'une gravure.

Les numismates parlent volontiers de gravure «lourde», de gravure de «meilleur style», de souci du détail ; on peut écarter par exemple la possibilité d'un atelier de faussaire par la qualité de la gravure.

La qualité de la gravure s'établit généralement en comparaison d'une autre monnaie, ou d'un autre groupe de pièces ou d'émissions, dont les éléments sont assez proches pour être comparés. Pour le reste, le jugement porté par le numismate sur une monnaie est souvent lié à une mode passagère, influencée par la vision de son temps, on en prendra comme exemple l'intérêt plus prononcé des collectionneurs ces dernières années pour les monnaies archaïques, aux lignes plus épurées, au détriment des monnaies hellénistiques au style peut-être trop raffiné. Mais, comme les historiens de l'art arrivent à le faire pour la peinture ou la sculpture, les spécialistes de la monnaie devraient être capables de s'accorder sur la qualité de la gravure d'un coin, à trouver des mesures communes pour juger de la qualité ou non du travail du graveur.

Il serait trop long ici de vouloir définir ces critères, je rappellerai simplement que mon jugement s'appuiera plus volontiers sur la qualité de la main de l'artisan

13. L'alliage d'une monnaie achetée dans le commerce et analysée lors de notre thèse contenait presque $50 \%$ de plomb (monnaie BAB 14).

14. Les expérimentations menées à Melle ces dernières années ont montré que la déformation du bronze au plomb était presque impossible. Sous le poids du marteau le métal se délite. 
plutôt que sur sa volonté de création, de modelage d'un type. Il me semble que le graveur doit être avant tout considéré comme un artisan dont la capacité première est de reproduire avec le plus d'exactitude possible le type décidé par l'autorité émettrice, que ce soit un roi, une cité ou tout autre type de gouvernement. D'ailleurs, un des critères de mesure de cette qualité semble bien être le temps passé à l'exécution du coin ${ }^{15}$; il peut être significatif de l'intérêt porté par l'autorité à l'émission ou de l'empressement à obtenir des monnaies dans un climat politique troublé.

Le monnayage des Ptolémées présente un grand nombre de pièces, d'or, d'argent ou de bronze, composées d'un alliage monétaire de très bonne qualité et dont on peut affirmer que les coins utilisés pour les frapper ont été soignés. C'est le cas pour la majorité des monnaies en métal précieux.

Par exemple, l'ensemble des pièces en or montre une gravure tout à fait maîtrisée et relativement délicate. Sur les mnaieia d'Arsinoé, ces pièces de 27,60 g frappées après la mort de la reine philadelphe, les traits sont précis et c'est le cas pour toute la série des premières mnaieia (celle dont les coins de droit sont numérotés avec des lettres), dont la composition est extrêmement régulière, aux alentours de $99 \%$ de fin (figure 5). On appréciera, ou pas, le côté un peu chargé du portrait de Ptolémée III émis par son fils (figure 6). Quoi qu'il en soit, la qualité de la gravure ne peut que difficilement être critiquée, pas plus que la qualité de la composition métallique, elle aussi proche de $99 \%$ de fin pour cette série.
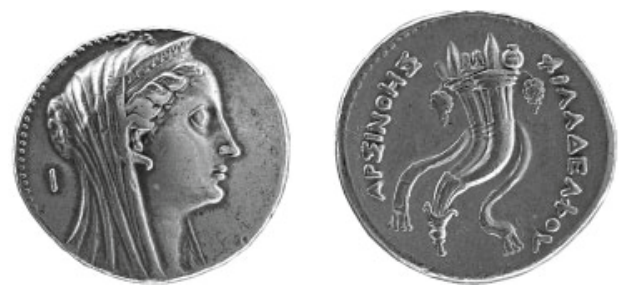

Figure 5 - Mnaieion en or, BnF, Fonds général nº 311.
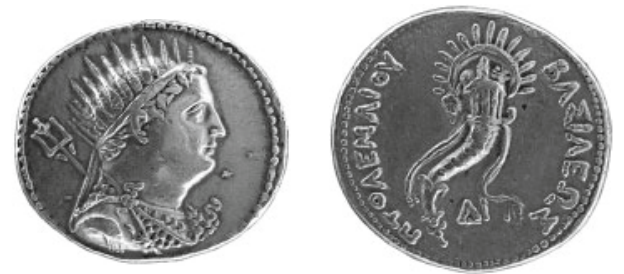

Figure 6 - Mnaieion en or, BnF, Fonds général nº 329.

15. Sur ce sujet, on prendra note des informations délivrées par l'étude du monnayage de bronze lagide par Bernard Bouyon, maître graveur de la Monnaie de Paris : B. Bouyon, G. DePEYrot, J.-L. DesniER, Systèmes et technologie des monnaies de bronze : $4^{e}$ s. avant J.-C.-3e s. après J.-C, Wetteren, Belgique, 2000, p. 16, mais aussi, de façon plus générale, B. BouYon, Études de gravure monétaire de l'Italie Grecque archaïque à l'Europe du $17^{e}$ siècle, Wetteren, Belgique, 1998. 
De manière générale, la qualité de la gravure lors du $\mathrm{III}^{\mathrm{e}}$ siècle était excellente, tout comme l'était la qualité de la composition métallique. C'est le cas pour l'or mais le constat est le même en ce qui concerne les monnaies d'argent et de bronze. Cela n'a rien de surprenant de retrouver une gravure de qualité équivalente sur les coins qui ont servi à frapper les pièces des trois métaux puisque les graveurs étaient peut-être les mêmes. On peut le penser pour l'argent et pour l'or, les mêmes signes apparaissant dans le creux d'une mèche derrière l'oreille du souverain sur les portraits de Ptolémée I $^{\text {er16. }}$.
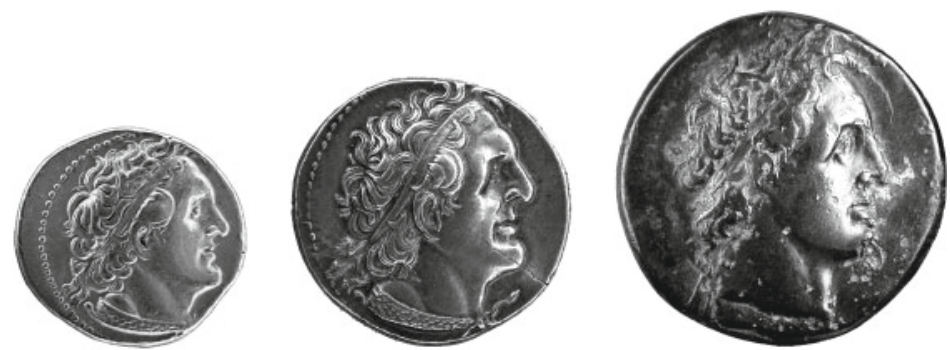

Figure 7 - Portraits de Ptolémée I ${ }^{\text {er }}$, en or (Svoronos 181, BnF, Fonds général nº14), en argent (Svoronos 201, BnF, Coll. De Luynes 3555) et en bronze

(Svoronos 549, Musée numismatique d'Athènes Z.4860).

La parenté stylistique est moins flagrante pour le monnayage de bronze même si la gravure de la monnaie représentée ci-dessus est également de bonne qualité. On notera simplement qu'il s'agit d'un triobole de la deuxième série (c.301-261) - une variété connue à très peu d'exemplaires - mais qui présente l'avantage d'être frappé au droit du portrait de Ptolémée I ${ }^{\mathrm{er}}$ comme les monnaies d'or et d'argent, ce qui facilite évidemment la comparaison.

Nous avons vu que certaines mnaieia au K présentaient une composition où entrait un pourcentage relativement important d'argent et/ou de cuivre. Il en existe plusieurs exemples au Cabinet des médailles comme, par exemple, une monnaie issue de la collection Beistègui (figure 8) qui contient 6,6\% d'argent et $1,29 \%$ de cuivre. Au contraire, d'autres de ces monnaies, comme celle de la figure 9 , un autre mnaieion au $\mathrm{K}$, ont une teneur en fin de près de $99 \%$. Il est pourtant impossible de saisir une différence importante de style entre ces deux monnaies. On ne peut donc pas mettre ici en relation le portrait empâté de la reine avec la qualité de l'alliage monétaire.

16. L'attribution du delta et des autres lettres et signes apparaissant derrière l'oreille du souverain à des graveurs a été remise en cause récemment par C. LORBER, A revised chronology for the coinage of Ptolemy I, NC, 2005, p. 45-64. Si le signe présent derrière l'oreille du roi ou bien sur l'égide d'Alexandre sur certaines pièces ne peut être le signe distinctif d'un seul graveur, nous avons tendance à penser qu'il peut être la marque d'un atelier de gravure ou d'une unité de production des coins. Il ne peut s'agir que d'une marque de contrôle dont la vérification prend place avant le début de la frappe des monnaies, la marque s'estompant très vite dès que le coin est utilisé. 

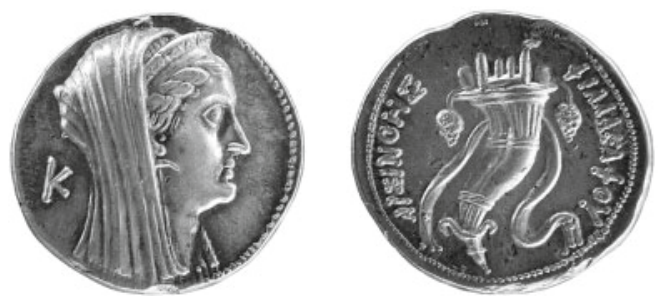

Figure 8 - Mnaieion en or (Svoronos 1498-9, BnF, Coll. Beistègui 48).
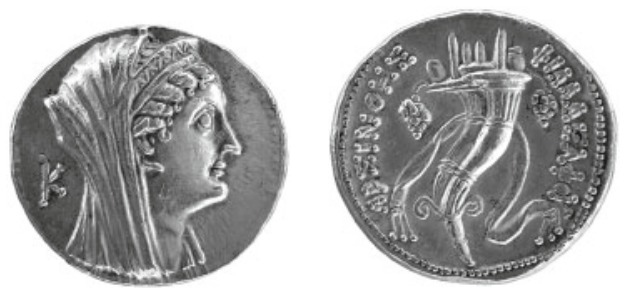

Figure 9 - Mnaieion en or (Svoronos 1498-9, BnF, Coll. Armand Valton 565).

À la fin de la période lagide, la qualité de la gravure des coins monétaires se détériore nettement même si certaines monnaies présentent encore quelques belles effigies. Cette monnaie frappée par Cléopâtre par exemple (figure 10) ne constitue pas le fleuron artistique du portrait lagide ; on sait en outre que ces monnaies ne contiennent que $30 \%$ d'argent dans leur alliage. Pourtant, l'exécution de la gravure des monnaies frappées quelques années auparavant par Ptolémée XII (figure 11), qui étaient constituées d'une teneur en fin beaucoup plus importante, souvent au-dessus de $90 \%$, n'est pas beaucoup plus remarquable que celle qui a suivi.
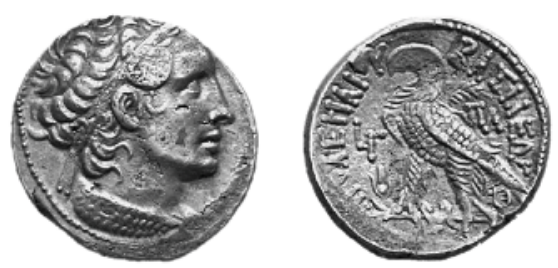

Figure 10 - Tétradrachme de Cléopatre VII, daté de 50/49

(Svoronos 1818, Dmitry Markov Coins \& Medals, Auction 11 Lot 174).
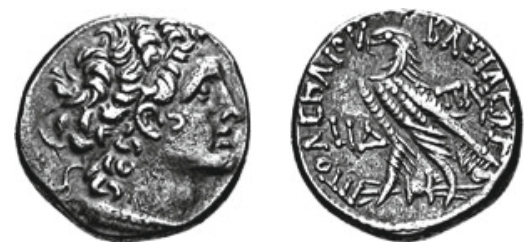

Figure 11 - Tétradrachme de Ptolémée XII, daté de 68/7 (Svoronos 1861, Ponterio \& Associates, Sale 146 Lot 1327). 
Dans la production des monnaies de bronze au contraire, on observe, avec la réforme de Cléopâtre (figure 13), l'apparition de monnaies réalisées beaucoup plus soigneusement que la plupart des exemplaires aux deux aigles qui ont envahi l'Égypte pendant près d'un siècle, dès le dernier quart du II ${ }^{\mathrm{e}}$ siècle. Les fouilles du Centre d'Études Alexandrines en ont fourni quelques centaines d'exemplaires (figure 12). Sans qu'il soit très avantageux, le portrait de la reine est précis et bien exécuté ; cette indéniable amélioration de la gravure dénote un regain d'intérêt de l'autorité pour la frappe des monnaies de bronze.
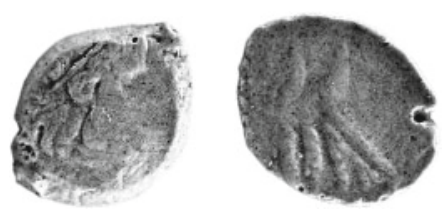

Figure 12 - Monnaie aux deux aigles de la série 9

(Svoronos 1426, monnaie Consulat nº 91, CEAlex).
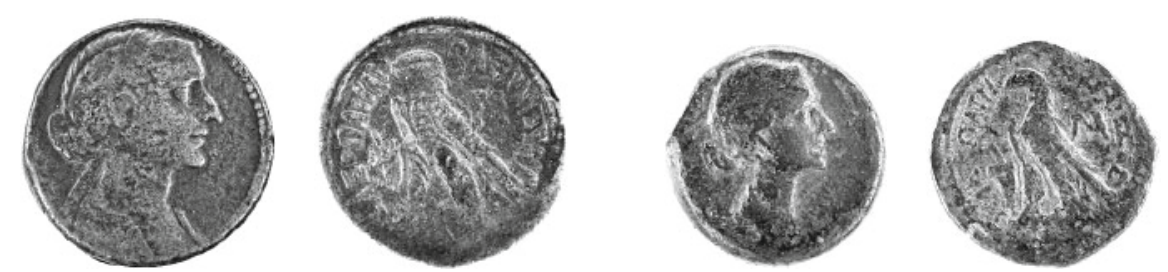

Figure 13 - Monnaies de Cléopatre VII, série 10 (c. 40-31), valant 80 drachmes (Svoronos 1871, BnF, Fonds général 467) et 40 drachmes (Svoronos 1872, BnF, Fonds général 474).

Mais d'autres types de monnaies brouillent encore un peu plus les pistes : il s'agit des monnaies moulées. D'une manière générale, les monnaies lagides moulées mises au jour lors de fouilles archéologiques sont très endommagées : la surface est craquelée, la tranche est fendue, autant de signes qui permettent de confondre le faussaire. Lors de l'expertise des monnaies de fouilles, ce type de monnaie offre l'avantage de présenter un faciès assez régulier : une cavité centrale au droit ainsi qu'au revers et un diamètre de $20 \mathrm{~mm}$. Ces caractéristiques permettent au numismate de les identifier sans doute possible et de les enregistrer dans la série 9, la série des monnaies aux deux aigles. Mais alors que nous pensions que cette technique était limitée à la production des monnaies aux deux aigles ${ }^{17}$, l'étude des monnaies du CEAlex nous a amené à revoir notre jugement puisqu'une monnaie de la série 7 (datée approximativement de 150 à

17. O. Picard, op.cit. n. 12. 
120), à la tête d'Isis, trouvée dans les fouilles du patriarcat orthodoxe, non loin de la rue Fouad, à Alexandrie, portait également les stigmates de ces monnaies moulées (figure 3, p. 99). La présence d'un gros nodule de plomb ne permet aucun doute ; cette monnaie, où l'on ne voit que très difficilement les contours de la tête d'Isis, a bien été moulée. On peut même dire que cette monnaie a circulé puisqu'elle a été découverte dans un quartier d'habitat de la ville.

À côté de cet exemplaire dans un état que l'on qualifiera de médiocre, il existe d'autres monnaies en meilleur état. Sur la monnaie provenant du trésor des bains de Karnak (figure 4, p. 99) ${ }^{18}$, malgré le gros nodule de plomb qui entache un peu la pièce et masque le menton de la déesse, la qualité de la gravure ici n'est pas désagréable. De nombreux exemplaires d'une plus grande qualité encore se trouvent en abondance dans une série de trésors monétaires enfouis au $\mathrm{II}^{\mathrm{e}}$ siècle $^{19}$. Sur ces monnaies, les traces de moulages sont moins visibles que sur les deux précédents exemples mais elles ne font aucun doute, il s'agit principalement des craquelures sur la surface, un des signes distinctifs des monnaies moulées.

Lorsque le moulage est de bonne qualité, la monnaie moulée peut donc être d'une gravure plaisante puisqu'elle reproduit en tous points la monnaie matrice que l'on choisissait certainement pour ses qualités esthétiques. Il en résulte une difficile distinction entre deux catégories : monnaies moulées de bonne ou de mauvaise qualité. Les trésors et les monnaies de fouilles procurent donc un large spectre de ces monnaies, de la plus soignée à la plus médiocre.

Cette distinction est d'autant plus difficile à faire qu'à l'intérieur des trésors, et c'est là le principal enseignement de ces trouvailles monétaires, les monnaies moulées et les monnaies frappées se mélangent. C'est le cas dans un trésor conservé au Musée égyptien du Caire (le trésor de Tell Nowa, $\mathrm{n}^{0}$ d'inventaire 2775), où l'on trouve des monnaies à la tête d'Isis d'un poids et d'un diamètre équivalents, mais de deux variétés différentes (Svoronos 1234 et 1384) ${ }^{20}$. Les analyses pratiquées à Orléans nous ont montré qu'une variété était caractérisée par une forte teneur en plomb (Svoronos 1384) alors que l'alliage des monnaies de l'autre variété (Svoronos 1234) n'en contenait que très peu. La conclusion s'impose : la composition métallique (donc la valeur intrinsèque) des monnaies de bronze n'avait pas d'effet sur l'utilisation des pièces, au moins à cette période.

Lorsqu'on cherche à mettre en relation la qualité de la gravure et le contenu métallique de la monnaie, les monnaies moulées en bronze ne sont pas les seules à poser problème. Les collections de monnaies d'argent comprennent également des pièces produites de façon frauduleuse : les monnaies fourrées.

18. M. Boraik, Th. Faucher, Le trésor des bains de Karnak, Cahiers de Karnak, 13, à paraître. 19. Trésor de Fouad, trésor des bains de Karnak et trésor de Tell Nowa.

20. Th. Faucher, Le trésor de Tell Nowa, BIFAO, 109, 2009, p. 105-134. 
Nous avons la chance de disposer au Musée gréco-romain d'Alexandrie certainement de la plus grande collection de monnaies lagides (c'est vrai pour l'argent et le bronze, ce n'est pas le cas pour l'or) mais aussi d'une série de quelques centaines d'alexandres. À l'occasion de la restauration des pièces en argent, les restauratrices disposant seulement d'éponges abrasives en raison du manque de moyens, un certain nombre de monnaies fourrées sont apparues, dévoilant l'âme de cuivre que cachait une mince pellicule d'argent (figure 14).
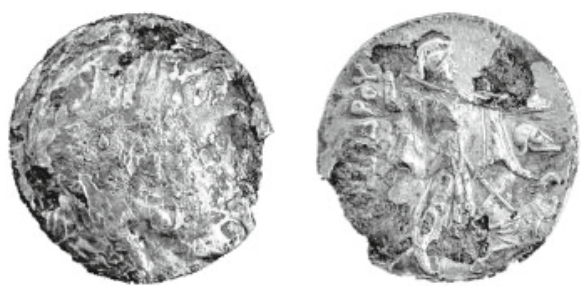

Figure 14 - Tétradrachme de Ptolémée I ${ }^{\text {er }}$

(Svoronos 162, $\mathrm{n}^{\circ}$ Alex 8-9 49).

Dans cette collection plusieurs monnaies fourrées ont ainsi été décelées. S'il est impossible de fournir un nombre exact pour l'instant, puisque seuls les alexandres et les monnaies de Ptolémée $\mathrm{I}^{\mathrm{er}}$ ont été étudiés en détail, cela représente une part non négligeable de l'ensemble. Il est loisible d'imaginer qu'une part encore plus grande de ces monnaies fourrées n'a pu être décelée. L'étude de coins n'a pas encore été menée, mais on s'attend à ce que les coins qui ont servi à la frappe de ces monnaies soient les mêmes que ceux qui frappaient les monnaies «officielles» et qu'apparaissent dès lors des liaisons de coins entre ces deux types de monnaies.

On pourrait multiplier les exemples montrant que la qualité de la gravure n'est pas toujours le signe d'une monnaie faite d'un alliage de qualité ; les nombreuses analyses de composition métallique menées au centre Ernest-Babelon depuis des années seraient là pour en témoigner. On prendra pour seul témoignage, l'un des plus significatifs : il s'agit des chouettes athéniennes.

Avec les analyses de J. Diebolt et de J.-N. Barrandon effectuées sous l'œil expert de $\mathrm{M}^{\mathrm{me}}$ Nicolet, nous avons appris que les chouettes athéniennes étaient faites du meilleur alliage ${ }^{21}$. On a d'ailleurs voulu identifier les imitations procheorientales par leur composition métallique.

21. Voir notamment J. Diebolt et H. Nicolet-PierRe, Recherches sur le métal de tétradrachmes à types Atheniens, $R S N, 1977$, p. 79-91. Pour un bilan de toutes les analyses menées à ce sujet, voir dernièrement : Chr. Flament, L'argent des chouettes. Bilan de l'application des méthodes de laboratoire au monnayage athénien tirant parti de nouvelles analyses réalisées au moyen de la méthode PIXE, $R B N, 2007$, p. 9-30. 

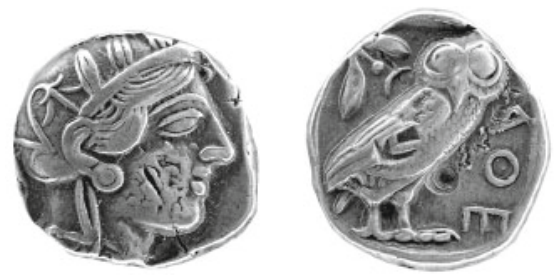

Figure 15 - Chouette athénienne, $\mathrm{Au}: 0,19 \%, \mathrm{Cu}: 1,85 \%$

(BnF, R2700).
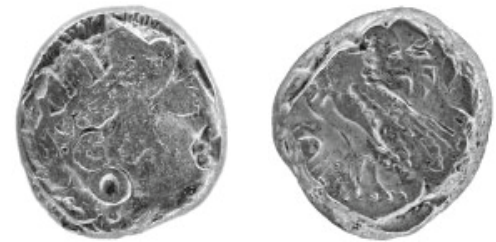

Figure 16 - Imitation ? chouette athénienne, $\mathrm{Au}: 1,10 \%, \mathrm{Cu}: 8,10 \%$

(BnF, R2708).

Si l'on sait maintenant que la grande majorité des chouettes ont été frappées à Athènes et qu'une partie importante des soi-disant «imitations» doit maintenant être réattribuée à l'atelier d'Athènes, il est difficile d'identifier avec certitude les imitations ${ }^{22}$. Pourtant, on a imité ces chouettes, les monnaies frappées par Mazakès et Sabakès en Égypte sont là pour le prouver, sans évoquer toutes les imitations proche-orientales. Mais il ne faudrait pas croire que les imitations soient caractérisées par une pauvre gravure et les monnaies bona fide par des traits gracieux. Il suffit pour cela de comparer quelques superbes exemplaires issus d'ateliers orientaux et des monnaies athéniennes dont la frappe (et la gravure) a certainement pu être hâtive, selon la nécessité pour les autorités de produire plus ou moins rapidement une grande quantité de pièces.
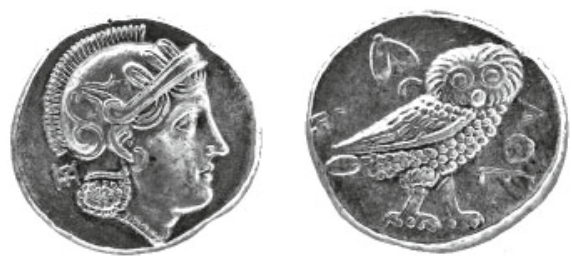

Figure 17 - Imitation orientale d'une chouette, Bactriane?

(BnF, Fonds général 1991_5 $)^{23}$.

22. Chr. Flament, Le monnayage en argent d'Athènes : de l'époque archaïque à l'époque hellénistique (c. 550-c. 40 av. J.-C.), Louvain-la-Neuve, 2007.

23. H. Nicolet-Pierre, Monnaies grecques trouvées en Afghanistan, RN, 1973, p. 35-42. 

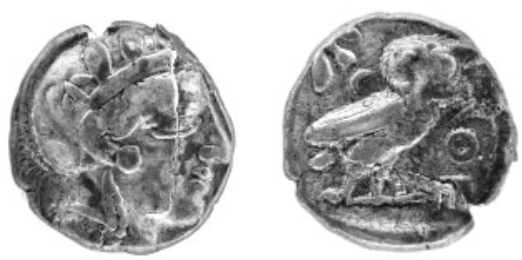

Figure 18 - Chouette athénienne bona fide

(BnF, Fonds général Y28153).

Que faut-il en conclure? Nous avons pu voir que certaines monnaies dont l'alliage est pauvre gardent tout de même une certaine grâce alors que des pièces avec une teneur en fin très élevée ont pu être frappées avec des coins dont le graveur aurait certainement mérité d'être sanctionné. Mais les raisons d'un traitement rapide de la gravure peuvent avoir de multiples explications. L'historien veut souvent y voir des troubles politiques mais la piètre qualité de certaines émissions devrait pouvoir s'expliquer par des événements macroéconomiques ou certaines contingences sur lesquelles nous n'avons aucun renseignement : pénurie de main d'œuvre, besoin urgent de produire des monnaies, désintérêt des autorités, absence de tradition monétaire, etc. Quoi qu'il en soit, les éclairages divers apportés par les monnaies lagides, qui reflètent à n'en pas douter les phénomènes monétaires des ateliers monétaires de la Méditerranée orientale à l'époque grecque, obligent à la plus grande prudence. Pour une même pièce, la qualité esthétique de la gravure et la qualité de la composition métallique diffèrent souvent, de sorte que notre jugement d'un monnayage ne devrait s'établir qu'avec la connaissance de ces deux paramètres. 